\title{
Kinder mit atopischer Dermatitis schulen, aber wie?
}

Der chronischen Erkrankung eines Kindes fühlt sich oft die ganze Familie hilflos ausgeliefert. Schulungsprogramme - z. B. für Kinder mit atopischer Dermatitis - können dazu beitragen, Betroffene bei der Krankheitsbewältigung zu unterstützen und auch die therapeutischen Langzeiterfolge zu verbessern, sagte PD Dr. Doris Staab vom Zentrum für Kinder- und Jugendmedizin der Charité, Berlin.

Während frühere Programme auf die Information des Patienten, die Verbesserung der Compliance und das praktische Training abzielten, stehe heute das „Empowerment" im Vordergrund. Ziel sei der verantwortungsbewusste, mündige Patient, so Staab. Es sei zudem internationaler Konsens, dass
Schulungen ein wichtiger Beitrag zur Therapie der atopischen Dermatitis sind. Umfang und Methoden verschiedener Konzepte differieren jedoch stark, sodass ein Vergleich kaum möglich sei [Stalder SF et al. 2013; 30: 329-34].

In Deutschland gut etabliert ist ein standardisiertes Programm der Arbeitsgemeinschaft Neurodermitisschulung e. V. (AGNES). Die Gruppenschulung für je sechs bis acht Familien umfasst sechs zweistündige Einheiten, die von einem interdisziplinären Team aus Pädiatern, Dermatologen, Psychologen und Ernährungsberatern durchgeführt werden. Neben medizinischen Informationen sind Hautpflege, Behandlungsmöglichkeiten, der Umgang mit
Juckreiz, Ernährungsfragen und die Bewältigung des Alltags wesentliche Inhalte. Die German Atopic Dermatitis Intervention Study (GADIS), die das Programm bei 823 Kindern und Jugendlichen evaluiert hat, konnte zeigen, dass die Intervention die Schwere der Erkrankung, den Umgang mit Juckreiz und die Lebensqualität verbessern kann [Staab D et al. BMJ 2006; 332: 933-8]. Schulungsprogramme sollten daher als essenzieller Bestandteil der Therapie in allen Leitlinien etabliert werden, forderte Staab. Angelika Bauer-Delto

Staab D. Atopic dermatitis. Workshop 15 "Educating the child with allergies"

\section{Anaphylaxie-Risiko bei Operationen senken}

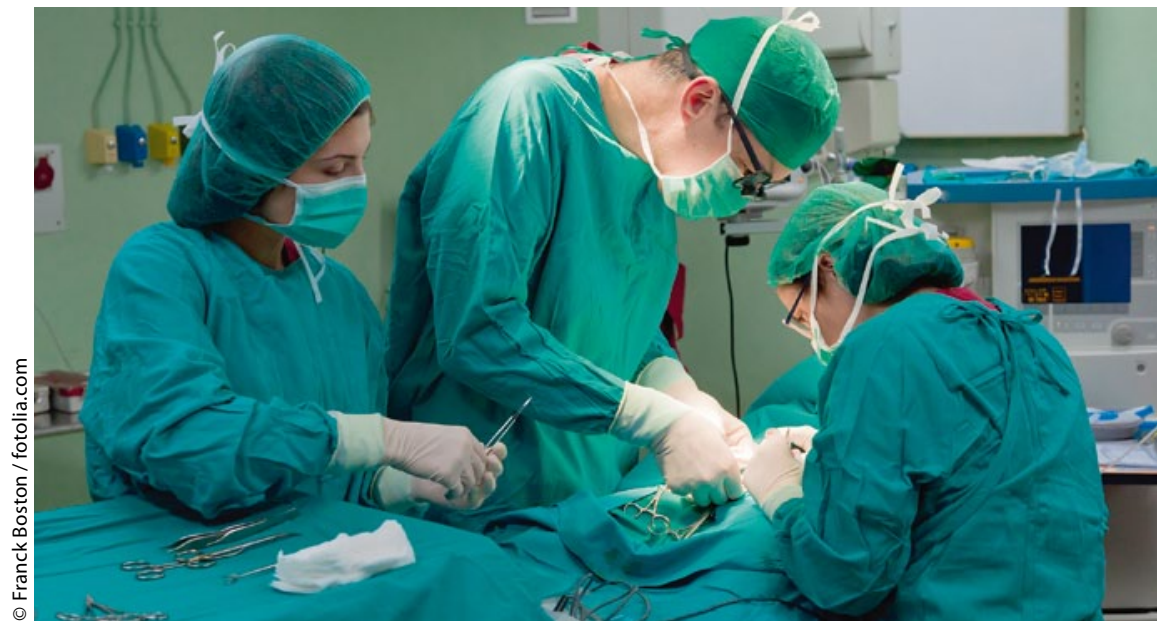

In einer von 10.000 Operationen kommt es während des Eingriffs zu Arzneimittelreaktionen.

Die Häufigkeit allergischer Reaktionen während eines operativen Eingriffs wird bislang unterschätzt, sagte Prof. Dr. PaulMichel Mertes, Universitätsklinikum Straßburg. Französischen Daten zufolge liegt die Inzidenz IgE-vermittelter Reaktionen während einer Anästhesie bei 1:10.000 Eingriffen [Mertes PM et al. J Allergy Clin Immunol 2011; 128: 366-73]. Die häufigsten Auslöser sind Muskelrelaxanzien, gefolgt von Latex. Von zunehmender Bedeutung sind Antibiotika und auch Farbstoffe sowie nicht steroidale Antiphlogistika.
Besondere Vorsicht ist bei Patienten geboten, die bereits bei früheren Eingriffen allergische Reaktionen gezeigt haben oder bei denen Arzneimittelreaktionen aus der Vorgeschichte - beispielsweise auf Antibiotika - bekannt sind. Ebenso sollten bei Nahrungsmittelallergien auf Avocado, Kiwi oder Banane mögliche Kreuzreaktionen auf Latex berücksichtigt werden. Ein erhöhtes Risiko tragen auch Kinder, die häufig operiert werden müssen. Bei Kindern mit Spina bifida konnte gezeigt werden, dass die Vermeidung einer Latexexposition die Sensibilisie- rungs- und Allergierate auf einen Bruchteil senken und sogar anderen allergischen $\mathrm{Er}$ krankungen vorbeugen konnte [Blumchen K et al. Allergy 2010; 65: 1585-93]. Auch die Auswahl der Wirkstoffe während eines operativen Eingriffs kann das Allergierisiko reduzieren. So führen die Muskelrelaxanzien Vecuronium und Atracurium wesentlich seltener zu anapyhlaktischen Reaktionen als Recuronium [Sadleir $\mathrm{PH}$ et al. $\mathrm{Br}$ J Anaesth 2013; 110: 981-7].

Eine interessante Hypothese sei auch, dass Pholcodin die Sensibilisierung auf Muskelrelaxanzien erhöht, berichtete Mertes. Skandinavische Daten weisen darauf hin, dass in Ländern, in denen Pholcodin-haltige Hustenblocker vom Markt genommen wurden, die Zahl anapyhlaktischer Reaktionen auf Muskelrelaxanzien drastisch sank [Florvaag E et al. World Allergy Organ J 2012; 5: 73-8].

Für das praktische Management von Arzneimittelreaktionen während operativer Eingriffe stehen heute umfassende Leitlinien zur Verfügung [Mertes PM et al., J Investig Allergol Clin Immunol 2011; 21: 442-53]. Diese werden in der Praxis jedoch noch nicht ausreichend berücksichtigt, kritisierte Mertes. Um im Notfall richtig reagieren zu können, sei ein intensives Training für Anästhesisten unerlässlich. Angelika Bauer-Delto

Mertes P-M. Risk reduction in general anesthesia. Workshop 12 "Sleeping shock - perioperative anaphylaxis" 